\title{
A New System Reliability Optimization Model Based on Swapping Existing Components
}

\author{
Yuxiong Li $\mathbb{D},{ }^{1}$ Xianzhen Huang $\mathbb{D},{ }^{1,2}$ Xinong En $\mathbb{D},{ }^{1}$ and Pengfei Ding $\mathbb{D}^{1}$ \\ ${ }^{1}$ School of Mechanical Engineering and Automation, Northeastern University, Shenyang, 110819, China \\ ${ }^{2}$ Key Laboratory of Vibration and Control of Aero-Propulsion Systems Ministry of Education of China, Northeastern University, \\ Shenyang, 110819, China \\ Correspondence should be addressed to Xianzhen Huang; xzhhuang83@gmail.com
}

Received 9 August 2019; Revised 30 October 2019; Accepted 6 November 2019; Published 25 November 2019

Academic Editor: Xianming Zhang

Copyright (c) 2019 Yuxiong Li et al. This is an open access article distributed under the Creative Commons Attribution License, which permits unrestricted use, distribution, and reproduction in any medium, provided the original work is properly cited.

\begin{abstract}
Complex systems contain a large number of components, and in some cases, failure of one or more of these components can cause the entire system to fail. Replacing failed components with other functioning components properly in the original system can be an attractive way for improving system reliability. This paper proposes a new system reliability optimization model to achieve optimal component reliability and the ideal component-swapping strategy under a certain set of constraints. Furthermore, the survival signature is introduced to more efficient calculation of system reliability under various component-swapping cases, and an artificial bee colony $(\mathrm{ABC})$ algorithm with local search method for component swapping is applied to solve the optimization problem. Finally, numerical examples are presented to illustrate the optimization process.
\end{abstract}

\section{Introduction}

As science and technology continue to progress, the complexity and scale of systems are rapidly increasing. Moreover, to achieve diverse sophisticated functions, these complex systems must be capable of operating under severe conditions such as uninterrupted high loads and extreme temperatures. The high risk and uncertainties associated with extreme environments can lead to serious accidents resulting in huge economic losses and threats to human lives [1-4].

Two fundamental methods are commonly applied for enhancement of system reliability. The first way is to improve reliability of components (reliability allocation), and the second way is to provide parallel redundancy components (redundancy allocation) [5, 6]. Since effort for improvement usually requires resources, system reliability optimization aims to obtain the optimal component reliability level and redundancy level to maximize the system reliability within a certain amount of resources [7]. Multiple heuristic algorithms have been proposed to solve such a nonlinear programming problem, and excellent results are achieved [8-12]. In addition, other mathematical methods have also been presented. Caserta and Voss [13] translated the reliability redundancy allocation problem into a knapsack problem solved by the branch and cut algorithm. Hemmati et al. [14] introduced neural networks in reliability redundancy allocation. Birnbaum importance and reliability sensitivity are also applied as reference for system reliability optimization [15-17]. Besides, some studies have widened the research subjects of system reliability optimization, including phased mission system [18], complex network system $[19,20]$, and system with uncertainties [21, 22].

As is clear from the literature, the existing research studies tend to focus on developing algorithms with high efficiency and applying them to various systems. However, the basic model of system reliability optimization is still limited in fundamental patterns, i.e., reliability and redundancy allocation, which will increase the cost, volume, and weight of the entire system and do not always yield ideal results. Component swapping is a new effective strategy for system reliability enhancement through swap between failed component and functioning component in a system [23]. This method is attractive since it makes full utilization of the 
existing components in the original system to avoid unnecessary cost and ponderosity of the entire system. However, not enough attention is paid on component swapping in related research and the algorithms for the optimal swapping strategy are rarely studied. Therefore, in this paper, a system reliability optimization model based on component swapping is proposed. Survival signature is introduced for efficient reliability calculation, and artificial bee colony algorithm is applied for optimization.

The remainder of the paper is organized as follows: Section 2 provides a description of the component-swapping strategy for improving system reliability and introduces the survival signature for more efficient reliability calculations under different swapping cases; Section 3 introduces the component swapping-based system reliability optimization model and artificial bee colony (ABC) algorithm for optimization; Section 4 presents three numerical cases to illustrate the application of newly proposed reliability optimization model based on component swapping; Finally, some conclusions and directions for future work are presented in Section 5.

\section{Swapping between Components in the Original System}

In practical systems, certain components have a higher degree of importance than others, and failure of these components can cause the entire system to malfunction [24]. To make systems more resilient to possible breakdowns, failed components with higher importance can be replaced with other components in the original system of lower importance that are still functioning [23]. Consider a system with three components, as shown in Figure 1. Component 1 and 2 are of the same type, and failure of component 1 will lead to the failure of the entire system. If component 1 fails and is replaced by component 2 , the system can continue working.

For the purpose of discussion, the components to be replaced are called "target components" and the components to replace target components are called "spare components." The following conditions are defined in this paper:

(1) Each target component corresponds to one unique spare component; that is to say, the maximum number of swapping sets for $n$ components of same type is $n / 2$ or $(n-1) / 2$, for $n$ is an even or odd integer

(2) Swapping only occurs when the target component and the whole system fail

(3) Swapping only occurs between the same type of components

(4) The time required for component swapping is ignored

2.1. Efficient Reliability Analysis with Survival Signature under Component Swapping. For a system with $n$ components, the vector $\mathbf{x}=\left(x_{1}, x_{2}, \ldots, x_{n}\right)$ describes the working state of all components where $x_{i}=1$ or 0 denotes functioning or failure, respectively, of the $i$ th component. Then, the structure function of the system $\phi(x)$ can be mapped as an associated state vector $x$ with a value of 1 or 0 denoting functioning or failure of the system, respectively [25].

For example, the structure function of the system presented in Figure 1 can be expressed as

$$
\phi(\mathbf{x})=x_{1}\left[1-\left(1-x_{2}\right)\left(1-x_{3}\right)\right] .
$$

Obtaining the structure function of a complex system with multiple components requires a large amount of calculations [26]. Furthermore, the structure of the system changes after component swapping, which makes it difficult to describe the system using the structure function. To calculate the system reliability more efficiently under various component swapping cases, truth table and survival signature are introduced.

The reliability truth table is a matrix describing the working status of the components and the system itself using Boolean values [27]. For a system with $n$ components, the truth table is a matrix of $2^{n}$ rows and $n$ columns. Each row represents a single possible combination of component statuses, and the entire truth table contains all possible combinations of component statuses. Each row of the truth table also has a corresponding indicator with a value of 0 or 1 representing either the failure or functioning of the whole system based on the statuses of components. Together, all $2^{n}$ indicators make up the indicator vector of the system.

For a system with 3 components, as shown in Figure 1, the truth table and the indicator vector are presented in Table 1.

When component swapping is introduced, the new structure of the system can be expressed by modifying the truth table of the original system. Since swapping only occurs when a target component and the whole system have failed, rows with a system status indicator value of 1 should remain unchanged. For the rest of the truth table, all rows with the target component value of 0 and the spare component value of 1 are found, and the values of 0 and 1 in each row are swapped. If the system can function after the rows are modified, the corresponding indicator changes from 0 to 1. Thus, the modified truth table and indicator vector can clearly describe the structure of the system after introducing component swapping. For example, to describe the swapping case where component 2 replaces component 1 in the system presented in Figure 1, transformation of the truth table is illustrated in Figure 2.

After the truth table and indicator vector are obtained, the reliability of the system can be calculated using the survival signature, which is an effective tool for the reliability analysis of complex systems with multiple component types. The survival signature represents the conditional probability that a system can maintain normal operation while a certain number of components in the system are functioning normally [28, 29]. For a system 


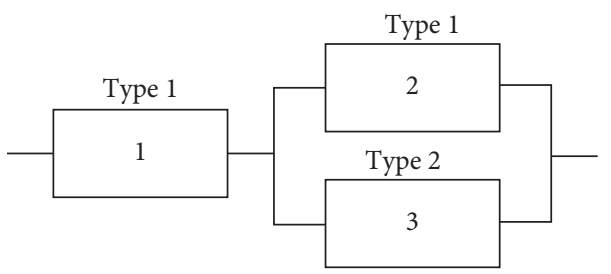

Before swapping

(a)

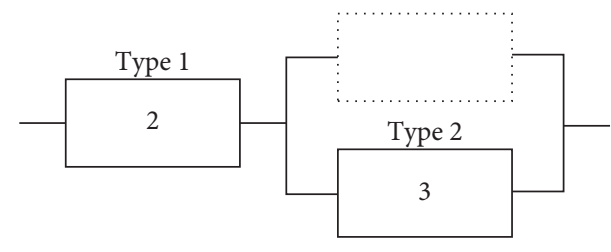

After swapping

(b)

FIGURE 1: Schematic illustration of component swapping. (a) Before the swapping. (b) After the swapping.

TABLE 1: Reliability truth table of the system with 3 components.

\begin{tabular}{lccc}
\hline Component 1 & Component 2 & Component 3 & System \\
\hline 0 & 0 & 0 & 0 \\
0 & 0 & 1 & 0 \\
0 & 1 & 0 & 0 \\
0 & 1 & 1 & 0 \\
1 & 0 & 0 & 0 \\
1 & 0 & 1 & 1 \\
1 & 1 & 0 & 1 \\
1 & 1 & 1 & 1 \\
\hline
\end{tabular}

\begin{tabular}{|c|c|c|c|c|c|c|c|c|c|c|c|c|c|}
\hline 1 & 2 & 3 & sys & & 1 & 2 & 3 & sys & & 1 & 2 & 3 & sys \\
\hline 0 & 0 & 0 & 0 & Areas & 0 & 0 & 0 & 0 & Update & 0 & 0 & 0 & 0 \\
\hline 0 & 0 & 1 & 0 & need & 0 & 0 & 1 & 0 & the systen & 0 & 0 & 1 & 0 \\
\hline 0 & 1 & 0 & 0 & modifying & & 0 & 0 & 0 & $\begin{array}{c}\text { indicator } \\
\text { vector }\end{array}$ & 1 & 0 & 0 & 0 \\
\hline 0 & 1 & 1 & 0 & & 1 & 0 & 1 & 0 & & 1 & 0 & 1 & 1 \\
\hline 1 & 0 & 0 & 0 & & 1 & 0 & 0 & 0 & & 1 & 0 & 0 & 0 \\
\hline 1 & 0 & 1 & 1 & 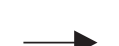 & 1 & 0 & 1 & 1 & $\rightarrow$ & 1 & 0 & 1 & 1 \\
\hline 1 & 1 & 0 & 1 & & 1 & 1 & 0 & 1 & & 1 & 1 & 0 & 1 \\
\hline 1 & 1 & 1 & 1 & & 1 & 1 & 1 & 1 & & 1 & 1 & 1 & 1 \\
\hline
\end{tabular}

FIgURE 2: Transformation of the truth table.

containing $K$ different types of components, the survival signature can be calculated as

$$
\Phi\left(l_{1}, l_{2}, \ldots, l_{K}\right)=\left[\prod_{k=1}^{K}\left(\begin{array}{c}
m_{k} \\
l_{k}
\end{array}\right)\right]^{-1} \times \sum_{x \in S_{l}} \phi(x)
$$

where $l_{1}, l_{2}, \ldots, l_{K}$ are the number of functioning components of each type, $m_{k}$ is the total number of components of type $k, \phi(\mathbf{x})$ is the structural function of the system, and $s_{i}$ denotes the set of all possible combinations of functioning components of each type, given the number of functioning components of each type $l_{1}, l_{2}, \ldots, l_{K}$. Since the truth table and indicator vector are already known for the system with component swapping, $\sum \phi(\mathbf{x})$ can be easily obtained by searching the matrix.

For the system shown in Figure 1, the survival signatures of the original system, $\Phi\left(l_{1}, l_{2}\right)$, and the system with component 1 swapped with component $2, \Phi_{s}\left(l_{1}, l_{2}\right)$, are presented in Table 2.
TABLE 2: Survival signature of the system with and without swapping.

\begin{tabular}{cccc}
\hline$l_{1}$ & $l_{2}$ & $\Phi\left(l_{1}, l_{2}\right)$ & $\Phi_{s}\left(l_{1}, l_{2}\right)$ \\
\hline 0 & 0 & 0 & 0 \\
0 & 1 & 0 & 0 \\
1 & 0 & 0 & 0 \\
1 & 1 & $1 / 2$ & 1 \\
2 & 0 & 1 & 1 \\
2 & 1 & 1 & 1 \\
\hline
\end{tabular}

The survival signature is essentially the conditional probability of system reliability; thus, the full probability equation can be used to derive the reliability of the system at a certain moment. Letting $C_{t}^{k}$ denote the number of functioning components of type $k$ at time $t$, the probability of the system functioning at time $t$ can be calculated as follows [28]:

$$
R_{S}(t)=P\left(T_{S}>t\right)=\sum_{l_{1}=0}^{m_{1}} \cdots \sum_{l_{K}=0}^{m_{K}}\left[\Phi\left(l_{1}, l_{2}, \cdots, l_{K}\right) \prod_{k=1}^{K} P\left(C_{t}^{k}=l_{k}\right)\right] .
$$

By using the cumulative distribution function (CDF) of type $k$ components, denoted by $F_{k}(t)$, the above equation can be further derived into the following form:

$$
\begin{aligned}
R_{S}(t)= & \sum_{l_{1}=0}^{m_{1}} \ldots \sum_{l_{K}=0}^{m_{K}}\left[\Phi\left(l_{1}, l_{2}, \ldots, l_{K}\right) \prod_{k=1}^{K}\right. \\
& \left.\cdot\left(\begin{array}{c}
m_{k} \\
l_{k}
\end{array}\right) F_{k}(t)^{m_{k}-l_{k}}\left[1-F_{k}(t)\right]^{l_{k}}\right] .
\end{aligned}
$$

2.2. Influence of Different Swapping Strategies on System Reliability. A bridge system consisting of 7 components of 2 types is presented in Figure 3 to illustrate all possible swapping cases. Letting $\left[a_{1}-b_{1}, \ldots, a_{n}-b_{n}\right]$ denote a certain swapping case in which target component $b_{i}$ is replaced with spare component $a_{i}(1 \leq i \leq n)$, all possible swapping cases for each type of component are listed in Tables 3 and 4 .

As shown in Tables 3 and 4, the total number of swapping cases for type 1 and type 2 components is 25 and 7 , respectively. Thus, the number of swapping cases for the whole system is $25 \times 7=175$. 


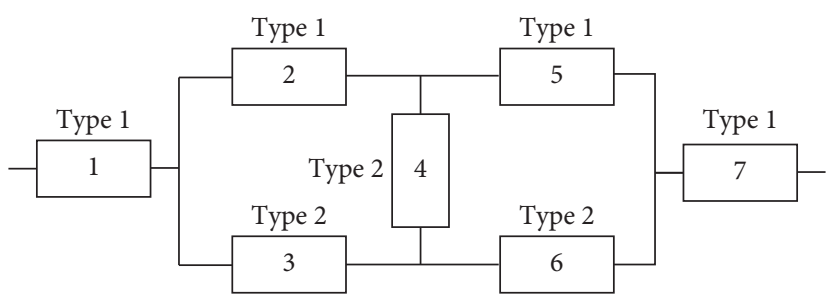

Figure 3: Bridge system with 7 components of 2 types.

TABle 3: All possible swapping cases for type 1 component.

\begin{tabular}{|c|c|c|}
\hline Number of swaps & Swapping cases & Number of cases \\
\hline 0 & {$[-]$} & 1 \\
\hline 1 & $\begin{array}{c}{[1-2],[1-5],[1-7],[2-5],[2-7],[5-7]} \\
{[2-1],[5-1],[7-1],[5-2],[7-2],[7-5]} \\
{[1-2,5-7],[1-7,5-2],[1-5,2-7],[2-1,5-7],[7-1,5-2]}\end{array}$ & 12 \\
\hline 2 & $\begin{array}{c}{[5-1,2-7],[1-2,7-5],[1-7,2-5],[1-5,7-2],[2-1,7-5]} \\
{[7-1,2-5],[5-1,7-2]}\end{array}$ & 12 \\
\hline
\end{tabular}

TABle 4: All possible swapping cases for type 2 component.

\begin{tabular}{lcc}
\hline Number of swapping & Swapping case & Number of cases \\
\hline 0 & {$[-]$} & 1 \\
1 & {$[3-4],[3-6],[4-6],[4-3],[6-3],[6-4]$,} & 6 \\
\hline
\end{tabular}

To illustrate the influence of different swapping strategies on system reliability, four cases are considered and discussed:

Case 1. Component 2 replaces component 1 in type 1 .

Case 2. Component 2 replaces component 5 in type 1.

Case 3. Component 2 replaces component 1 in type 1 and component 3 replaces component 4 in type 2 .

Case 4. Component 2 replaces component 1 in type 1 and component 4 replaces component 3 in type 2 .

Truth tables and indicator vectors for the original bridge system under four different swapping cases can be applied according to Section 2.1. The survival signature of the original system, $\Phi\left(l_{1}, l_{2}\right)$, and the survival signature of the system under four different swapping cases, $\Phi_{1}\left(l_{1}, l_{2}\right), \Phi_{2}\left(l_{1}\right.$, $\left.l_{2}\right), \Phi_{3}\left(l_{1}, l_{2}\right)$, and $\Phi_{4}\left(l_{1}, l_{2}\right)$, were calculated by equation (2), and the results are presented in Table 5. Rows with $\Phi\left(l_{1}\right.$, $\left.l_{2}\right)=\Phi_{1 \sim 5}\left(l_{1}, l_{2}\right)=0$ are omitted since these data do not affect the calculations.

The CDFs of each type of component in Figure 3 can be expressed as follows:

$$
\begin{aligned}
& F_{1}(t)=1-e^{-(t / 3)^{4}} \\
& F_{1}(t)=1-e^{-(t / 4)^{4}}
\end{aligned}
$$

Reliability curves of the original system and the system under four swapping cases were derived and are shown in Figure 4.
TABLE 5: Survival signature of the system under different swapping strategies.

\begin{tabular}{ccccccc}
\hline$l_{1}$ & $l_{2}$ & $\Phi\left(l_{1}, l_{2}\right)$ & $\Phi_{1}\left(l_{1}, l_{2}\right)$ & $\Phi_{2}\left(l_{1}, l_{2}\right)$ & $\Phi_{3}\left(l_{1}, l_{2}\right)$ & $\Phi_{4}\left(l_{1}, l_{2}\right)$ \\
\hline 2 & 2 & $1 / 18$ & $1 / 9$ & $1 / 18$ & $1 / 18$ & $2 / 9$ \\
2 & 3 & $1 / 6$ & $1 / 3$ & $1 / 6$ & $1 / 3$ & $1 / 3$ \\
3 & 2 & $1 / 3$ & $1 / 2$ & $5 / 12$ & $5 / 12$ & $2 / 3$ \\
3 & 3 & $1 / 2$ & $3 / 4$ & $1 / 2$ & $3 / 4$ & $3 / 4$ \\
4 & 0 & 1 & 1 & 1 & 1 & 1 \\
4 & 1 & 1 & 1 & 1 & 1 & 1 \\
4 & 2 & 1 & 1 & 1 & 1 & 1 \\
4 & 3 & 1 & 1 & 1 & 1 & 1 \\
\hline
\end{tabular}

As shown in Figure 4, component swapping considerably improves the reliability of the entire system, and a clear gap between improvements can be observed among the different swapping strategies. It is worth noting that more components were swapped in Case 3 compared to Case 1. However, a smaller improvement in reliability was observed for Case 3 because swapped components can affect other components, leading to disconnection of some pathways in some cases. For example, if components 1, 4, and 5 all fail at the same time, the system will not work properly and all of the failed components will need to be replaced (Figure 5).

The system structure after separately implementing swapping strategies 1 and 3 is shown in Figures 6 and 7, respectively.

In Case 1 , failed component 1 is replaced by component 2 and a connected path [2 36 6 7] is created, which allows the system to continue operating normally. However, in Case 3, component 4 is also replaced by component 3 in addition to swapping components 1 and 2 and no connected path is 


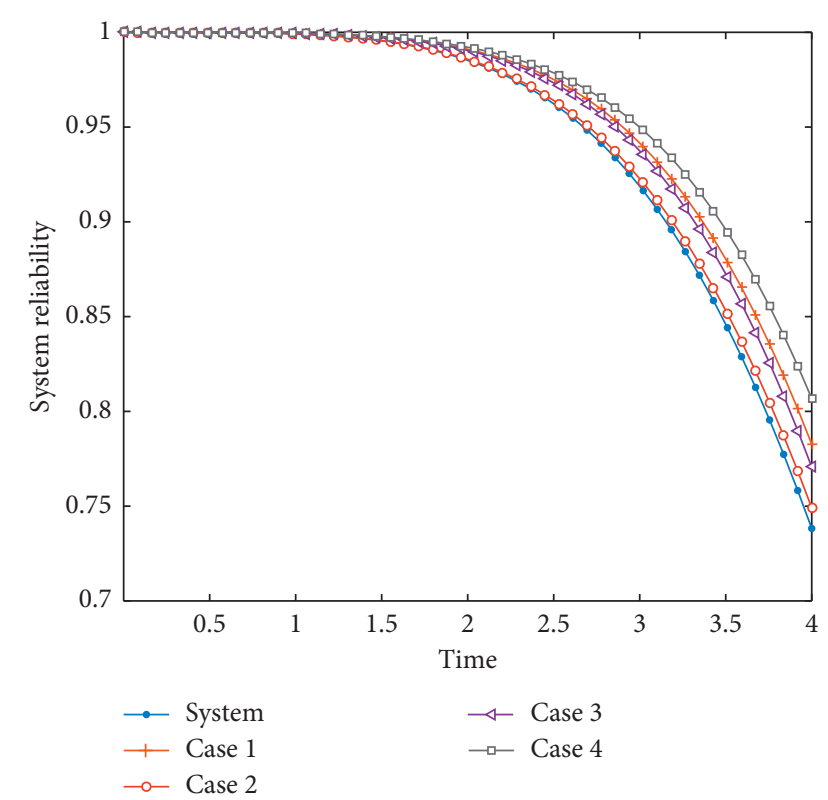

FIGURE 4: Reliability curves of the system under four different component-swapping cases.

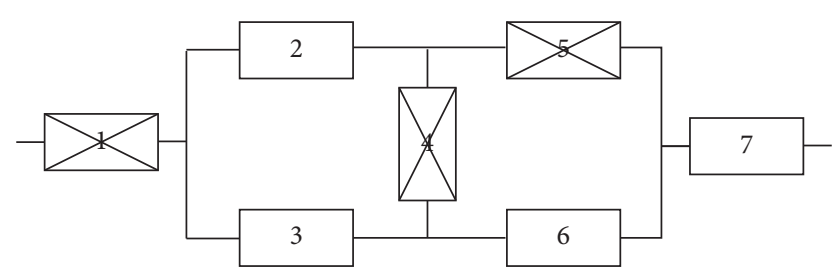

FIgURE 5: System with three failed components.

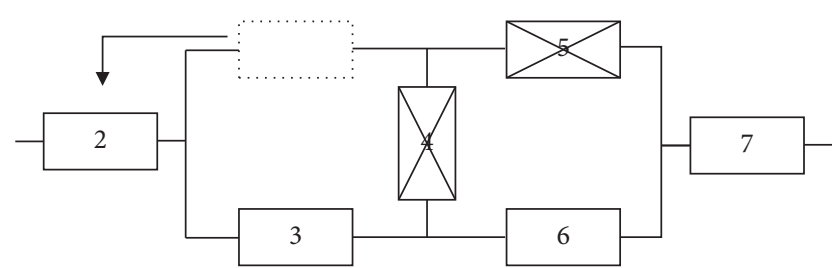

FIGURE 6: System structure after implementing swapping strategy 1.

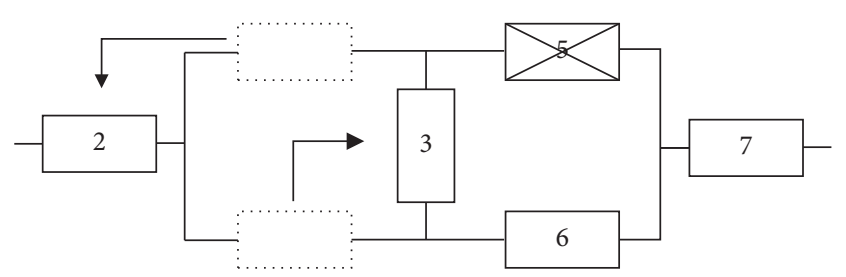

Figure 7: System structure after implementing swapping strategy 3.

created. Thus, the system cannot continue to function properly.

This can be explained by the Birnbaum importance [30]. The Birnbaum importance of a component can reflect the impact of component status on system reliability, which can be measured by

$$
\mathrm{BI}_{i}=\frac{\partial R_{\mathrm{S}}(t)}{\partial R_{i}(t)}=h\left(1_{i}, R(t)\right)-h\left(0_{i}, R(t)\right),
$$

where $R_{i}$ is the reliability of $i$ th component at time $t$ and $h\left(1_{i}\right.$, $R(t))$ and $h\left(0_{i}, R(t)\right)$ represent the reliability of the system with $i$ th component that is working and failed, respectively. The importance curves of component 3 and component 4 in the bridge system obtained by equation (6) are shown in Figure 8 .

As can be seen above, the importance of component 3 is always higher than that of component 4 during the working period, which means replacing component 4 with component 3 is not an effective strategy to improve system reliability.

With the increase in scale and complexity of the system, it becomes more complicated to determine the effective swapping strategy based on component importance. Therefore, a swapping-based system reliability optimization model is built and a heuristic algorithm is applied to achieve the optimal swapping strategy.

\section{Component Swapping-Based System Reliability Optimization}

Section 2 shows large variation in system reliability improvement depending on the component-swapping case selected. For complex systems with a large number of components, more feasible swapping strategies are available to choose from; therefore, new optimization approaches for these problems are necessary. In practice, many factors inevitably restrict swapping of components; thus, component swapping should be considered an optional way to improve system reliability under various constraints, instead of component redundancy. A new reliability optimization model can be constructed that searches for the optimal component reliability as well as the best componentswapping strategy to maximize reliability of the system under certain constraints, such as cost, weight, and volume.

3.1. Establishment of Swapping-Based Reliability Optimization Model. Consider a system comprised of $K$ different types of components with $m_{k}$ components of type $k(1 \leq k \leq K)$. Components of the same type share the same reliability, and swapping can only occur between the same component type. For ease of programming, the swapping strategy for components of type $k$ can be expressed by matrix $\mathbf{h}_{k}$ with $n$ rows and 2 columns:

$$
\mathbf{h}_{k}=\left[\begin{array}{cc}
x_{1} & y_{1} \\
x_{2} & y_{2} \\
\vdots & \vdots \\
x_{n_{k}} & y_{n_{k}}
\end{array}\right],
$$

where $x$ represents spare components of type $k, y$ represents target components of type $k$, and $n_{k}$ represents the number of component-swapping set in type $k$, which should not exceed $m_{k} / 2$ since each target component corresponds to 


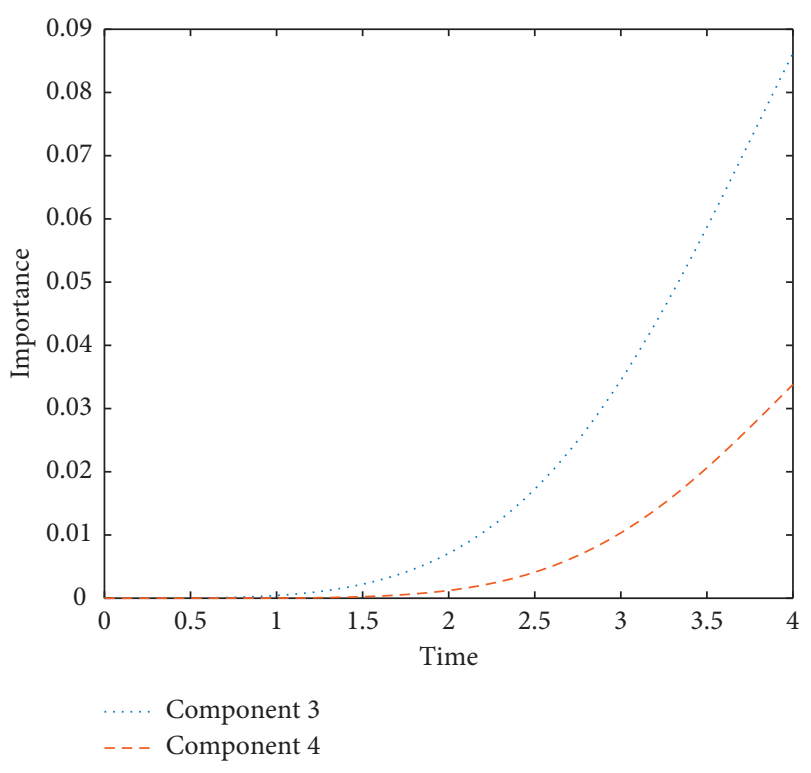

FIGURE 8: Importance of component 3 and component 4.

only one unique spare component. It should be noted that matrices can only describe swapping of a single component type; therefore, exchanging rows within the matrix will have no effect on the calculations. Moreover, if no type $k$ component is swapped, $\mathbf{h}_{k}$ is an empty matrix.

Taking the bridge system presented in Figure 3 as an example, for type 1 , component 2 replaces component 1 and component 5 replaces component 7 and for type 2, component 4 replaces component 3 . Then, the swapping strategy can be expressed using the following matrices:

$$
\begin{aligned}
& \mathbf{h}_{1}=\left[\begin{array}{ll}
2 & 1 \\
5 & 7
\end{array}\right], \\
& \mathbf{h}_{2}=\left[\begin{array}{ll}
4 & 3
\end{array}\right] .
\end{aligned}
$$

The survival signature of the system for a certain swapping case $\Phi_{h_{1}, h_{2}, \ldots, h_{K}}$ can be obtained from equation (2), and under a certain component reliability and certain swapping strategy, the system reliability can be expressed by equation (4). Therefore, the swapping-based system reliability optimization problem can be seen as a nonlinear mixed-integer programming problem for determining the optimal component reliability of each component type and the best component-swapping matrix for each. The mathematical model can be defined as follows:

$$
\begin{array}{cl}
\operatorname{maximum} \quad R_{s}(\mathbf{r}, \mathbf{h})=\sum_{l_{1}=0}^{m_{1}} \ldots \sum_{l_{K}=0}^{m_{K}}\left[\Phi_{\mathbf{h}_{1}, \mathbf{h}_{2}, \ldots, \mathbf{h}_{K}}\left(l_{1}, l_{2}, \ldots, l_{K}\right)\right. \\
& \left.\cdot \prod_{k=1}^{K} r_{k}^{l_{k}}\left(1-r_{k}\right)^{m_{k}-l_{k}}\right], \\
\text { subject to } \quad & g_{1}(\mathbf{r}, \mathbf{h})=V_{S}(\mathbf{r}, \mathbf{h})-V \leq 0, \\
g_{2}(\mathbf{r}, \mathbf{h})= & C_{S}(\mathbf{r}, \mathbf{h})-C \leq 0, \\
g_{3}(\mathbf{r}, \mathbf{h})= & W_{S}(\mathbf{r}, \mathbf{h})-W \leq 0,
\end{array}
$$

where $\mathbf{r}=\left[r_{1}, r_{2}, \ldots, r_{K}\right]^{T}$ is a variable vector containing the reliability of components in each type, $\mathbf{h}=\left\{\mathbf{h}_{1}, \mathbf{h}_{2}, \ldots, \mathbf{h}_{K}\right\}$ is a collection containing $K$ matrices representing the swapping strategy for components in each type, and $V_{s}(\cdot), C_{s}(\cdot)$, and $W_{\mathrm{s}}(\cdot)$ are the total volume, cost, and weight, respectively, as a function of the component reliability and swapping case.

Obviously, component swapping does not directly increase the weight and volume of the system. However, the movement of spare components to the target component position will undoubtedly bring some inconvenience to the process of swapping considering the weight and volume of the components. In order to reflect the limitation of the weight and volume of components on the number of swapping in the optimization model, the constraint functions $V_{\mathrm{s}}(\cdot)$ and $W_{\mathrm{s}}(\cdot)$ are defined as follows:

$$
\begin{gathered}
V_{\mathrm{S}}(\mathbf{r}, \mathbf{h})=\sum_{k=1}^{K}\left(v_{k} n_{k}^{3}+v_{k} m_{k}\right), \\
W_{\mathrm{S}}(\mathbf{r}, \mathbf{h})=\sum_{k=1}^{K}\left(\omega_{k} n_{k}^{3}+\omega_{k} m_{k}\right),
\end{gathered}
$$

where $n_{k}$ is the number of swapping sets in type $k$ components, i.e., the number of rows of matrix $\mathbf{h}_{k} ; w_{k}$ and $v_{k}$ are the weight and volume factors of type $k$ components; the additional terms in the constraint functions, $\omega_{k} m_{k}$ and $v_{k} m_{k}$, represent the volume and weight of the original components in the system. The cost function $C_{s}(\cdot)$ increases with component reliability and the number of swapping sets in the system and can be expressed as follows [7]:

$$
C_{S}(\mathbf{r}, \mathbf{h})=\sum_{k=1}^{K} \alpha_{k}\left(-\frac{T}{\ln \left(r_{k}\right)}\right)^{\beta_{k}}\left[m_{k}+n_{k}+\exp \left(\frac{n_{k}}{4}\right)\right],
$$

where $\alpha$ and $\beta$ are known constants representing the scaling factor and the shaping factor of the component, respectively, and $T$ is the mission time during which the system must function normally.

3.2. Penalty Guided Artificial Bee Colony Algorithm. Once a system reliability optimization model is established, the optimal solution can be quickly obtained through intelligent algorithms. In this paper, the artificial bee colony (ABC) algorithm is used [31, 32]. The ABC algorithm based on the forging behavior of honey bees can search over both feasible and infeasible space to find the best solution; thus, the optimal swapping strategy can be obtained from finite possible strategies after multiple iterations.

For the ABC algorithm to be capable of solving the constrained optimization problem, it is necessary to process constraints in the optimization problem and add them to the objective function as a penalty factor. The penalized optimization objective function can be expressed as [32] 


$$
R_{\mathrm{p}}= \begin{cases}-R_{s}, & \text { if } g_{1}(\mathbf{r}, \mathbf{h}) \leq 0, g_{2}(\mathbf{r}, h) \leq 0, g_{3}(\mathbf{r}, \mathbf{h}) \leq 0, \\ -R_{s}\left(\min \left\{\left[\frac{V}{V_{S}(\mathbf{r}, \mathbf{h})}\right],\left[\frac{C}{C_{S}(\mathbf{r}, \mathbf{h})}\right],\left[\frac{W}{W_{S}(\mathbf{r}, \mathbf{h})}\right]\right\}\right)^{\gamma}, & \text { otherwise, }\end{cases}
$$

where $R_{\mathrm{S}}$ is the system reliability obtained by using (9) and $\gamma$ is a positive amplification constant. A flowchart of the algorithm is presented in Figure 9. The main processes for solving the swapping-based reliability optimization problem using the $\mathrm{ABC}$ algorithm are as follows.

(1) Setting Parameters of the Algorithm

The maximum cycle number of the algorithm $M$, population of solutions $N$, and limit update number $L$ are set.

(2) Solution Initialization

The initial population is formed by randomly generating $N$ solutions. Reliability of components of type $k$ can be generated as follows [32]:

$$
r_{k}=r_{\min }+\operatorname{rand}[0,1]\left(r_{\max }-r_{\min }\right) \text {, }
$$

where $r_{\max }$ and $r_{\min }$ represent the upper and lower bounds of reliability, respectively.

The swapping matrices of the system are generated by stochastic extraction and random arrangement. First, a random integer $n_{k}$ is generated to determine the number of component pairs participating in swapping of type $k$ :

$$
n_{k}= \begin{cases}\operatorname{randi}\left[0, \frac{m_{k}}{2}\right], & \text { if } m_{k} \text { is even, } \\ \text { randi }\left[0, \frac{m_{k}-1}{2}\right], & \text { if } m_{k} \text { is odd }\end{cases}
$$

Then, $2 n_{k}$ elements are randomly selected from the set of type $k$ components and randomly arranged to form matrix $\mathbf{h}_{k}$. The process can be realized using the randperm function in MATLAB.

(3) Fitness Evaluation for Each Solution

To describe the degree of excellence of each solution, a fitness value is calculated using the following equation:

$$
\mathrm{fit}_{i}= \begin{cases}\frac{1}{R_{p i}+1}, & R_{p i} \geq 0, \\ \left|R_{p i}\right|+1, & R_{p i}<0,\end{cases}
$$

where $R_{p i}$ is the optimization result of the $i$ th solution calculated by equation (12).

(4) Local Search and Generating New Solutions

Let $\mathbf{X}_{i}=\left\{r_{i, 1}, \ldots, r_{i, K}, h_{i, 1}, \ldots, h_{i, K}\right\}$ be the $i$ th solution in the whole swarm population. To generate a new candidate solution $X_{i}^{\text {new }}$ nearby $X_{i}$, a variable index $z(1 \leq z \leq 2 K)$ is generated and solution $\mathbf{X}_{j}=\left\{r_{j, 1}, \ldots\right.$, $\left.r_{j, K}, h_{j, 1}, \ldots, h_{j, K}\right\}(i \neq j)$ is randomly selected. Except for the $z$ th variable, the rest of the newly generated solution is the same as the original solution $\mathbf{X}_{i}$.

If $z \leq K$, the variable to be modified is the reliability of type $z$ components, which can be generated as follows [32]:

$$
r_{i, z}^{\text {new }}=r_{i, z}+\phi\left(r_{i, z}-r_{j, z}\right)
$$

where $\phi$ is a random parameter between 1 and -1 ; thus, the new solution will be

$$
\mathbf{X}_{i}^{\text {new }}=\left\{r_{i, 1}, \ldots, r_{i, z}^{\text {new }}, \ldots, r_{i, K}, \mathbf{h}_{i, 1}, \ldots, \mathbf{h}_{i, K}\right\} .
$$

If $K<z \leq 2 K$, the variable to be modified is the swapping matrix for components of type $z-K$ which can be generated as follows:

$$
\mathrm{h}_{i, z-K}^{\text {new }}=\left[\begin{array}{l}
\mathbf{a}_{z_{1} \times 2} \\
\mathbf{b}_{z_{2} \times 2}
\end{array}\right] \text {, }
$$

where $\mathbf{a}$ is a matrix of $z_{1}$ rows and 2 columns, consisting of rows of the same content between matrix $\mathbf{h}_{i, z-K}$ and $\mathbf{h}_{j, z-K}$. For example, if $\mathbf{h}_{i, z-K}$ is [ $14 ; 25 ; 93$; 6 7] and $\mathbf{h}_{j, z-K}$ is $[93,68,14]$, then $\mathbf{a}$ can be formed as $[93,14]$. In addition, a matrix $\mathbf{b}$ with $z_{2}$ rows and 2 columns can be randomly generated using the method described in step 1: removing all elements of matrix a from components of type $z-K$, then randomly selecting $2 z_{2}$ elements from the remainder, and randomly arranging them to form rest of the matrix, where $z_{2}$ is a random integer index, generated as follows:

$$
z_{2}= \begin{cases}\operatorname{randi}\left[0, \frac{m_{z-K}}{2}-2 z_{1}\right], & \text { if } m_{z-K} \text { is even, } \\ \operatorname{randi}\left[0, \frac{m_{z-K}-1}{2}-2 z_{1}\right], & \text { if } m_{z-K} \text { is odd. }\end{cases}
$$

Thus, the new solution is

$$
\mathbf{X}_{i}^{\text {new }}=\left\{r_{i, 1}, \ldots, r_{i, K}, \mathbf{h}_{i, 1}, \ldots, \mathbf{h}_{i, z-K}^{\text {new }}, \ldots, \mathbf{h}_{i, K}\right\} .
$$

Then, the solutions are substituted into the objective function to calculate the fitness using equation (15). If the newly generated solution is better than the original one, the original is replaced with the new one; otherwise, the original solution is retained.

(5) Assign Onlooker Bees

Evaluate the whole swarm population and accept the solution with probability calculated as follows [32]: 


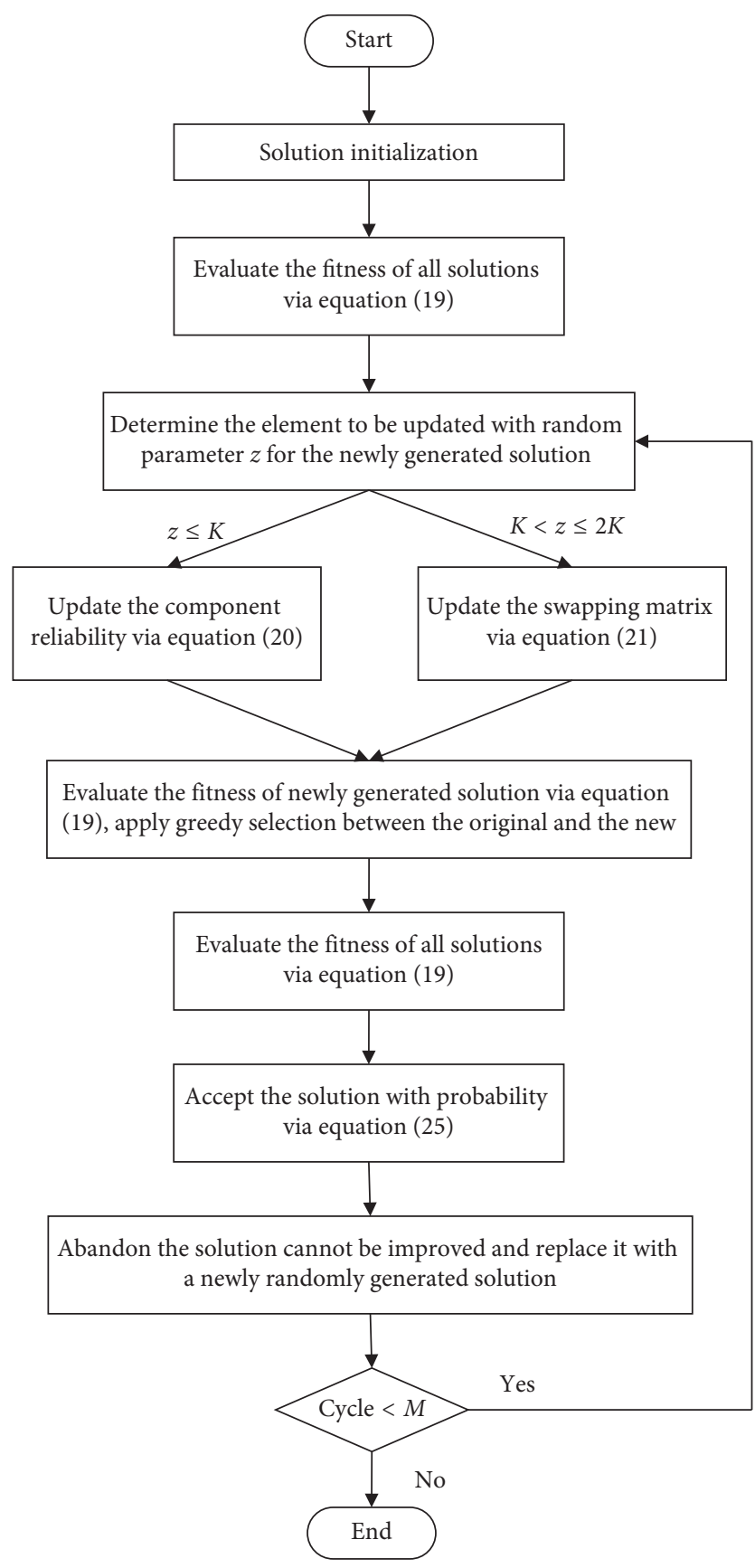

FIgURE 9: Flowchart of optimization algorithm.

$$
P_{i}=\frac{\mathrm{fit}_{i}}{\sum_{h=1}^{N} \mathrm{fit}_{h}},
$$

where fit is the fitness value of the solution reflecting the superiority of solution vector and $N$ is the total number of solutions of the whole swarm; moreover, the better the solution, the more likely it will be accepted.

(6) Determine the Abandoned Solution

If a solution is not accepted after $L$ cycles of update, the solution is abandoned and replaced with a new solution randomly generated using Step 1.
(7) Repeat the Aforementioned Steps for M Iterations

\section{Numerical Examples}

A swapping-based system reliability optimization model was established for two complex systems, and the ABC algorithm was applied to determine the optimal component reliability and best swapping strategy. Multiple calculations were carried out for each example to verify the stability of the algorithm.

Example 1. A system consisting of 9 components of 2 types is shown in Figure 10. Components 1, 2, 3, 5, and 8 are type 1 , and components $4,6,7$, and 9 are type 2 . Parameters used in the model are listed in Table 6. Assuming the failure time of components in the system obeys an exponential distribution, and failure rates of each component, type 1 and type 2 , at time $T$ are 0.05 and 0.07 , respectively.

The swapping-based reliability optimization mathematical model can be defined as follows:

$$
\begin{aligned}
\text { maximum } \quad R_{\mathbf{s}}(\mathbf{r}, \mathbf{h}) & =\sum_{l_{1}=0}^{4} \sum_{l_{2}=0}^{5}\left[\Phi_{h_{1}, h_{2}}\left(l_{1}, l_{2}\right) \prod_{k=1}^{2} r_{k}^{l_{k}}\left(1-r_{k}\right)^{m_{k}-l_{k}}\right], \\
\text { subject to } \quad g_{1}(\mathbf{r}, \mathbf{h}) & =\sum_{k=1}^{2}\left(v_{k} n_{k}^{3}+v_{k} m_{k}\right)-V \leq 0, \\
g_{2}(\mathbf{r}, \mathbf{h}) & =\sum_{k=1}^{2} \alpha_{k}\left(-\frac{T}{\ln \left(r_{k}\right)}\right)^{\beta_{k}}\left[m_{k}+n_{k}+\exp \left(\frac{n_{k}}{4}\right)\right]-C \leq 0, \\
g_{3}(\mathbf{r}, \mathbf{h}) & =\sum_{k=1}^{2}\left(\omega_{k} n_{k}^{3}+\omega_{k} m_{k}\right)-W \leq 0 .
\end{aligned}
$$

The maximum cycle number was set to 2000 , the population of solutions was set to 50, and the limit update number was set to 100 . Then, optimization was performed by applying the penalty guided ABC algorithm. The optimal component reliability and swapping strategy are shown in Table 7.

After multiple calculations, the optimal reliability at time $t=T$ was 0.90847271 and 0.86742572 for type 1 and type 2 components, respectively. The optimal swapping strategy for the system is as follows: component 8 is set to replace component 5 in type 1 ; component 7 is set to replace component 4 and component 9 is set to replace component 6 in type 2.

The survival signature of the original system, $\Phi\left(l_{1}, l_{2}\right)$, and the survival signature under the optimal swapping strategy, $\Phi_{s}\left(l_{1}, l_{2}\right)$, were calculated using equation (2), and the results are presented in Table 8 . Rows with $\Phi\left(l_{1}\right.$, $\left.l_{2}\right)=\Phi_{s}\left(l_{1}, l_{2}\right)=0$ are omitted since these data have no effect on the calculations.

Birnbaum importance of components is listed in Table 9 to examine the effectiveness of the swapping strategy. As can be seen from Table 9, the importance of target components is higher than that of spare components in each type, which 


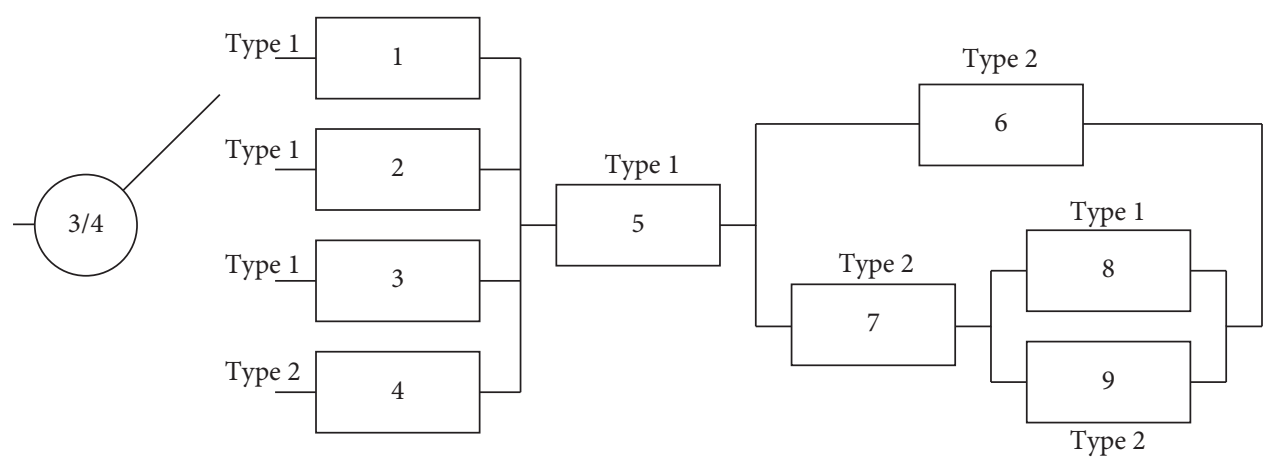

Figure 10: System with 9 components of 2 types.

Table 6: Parameters used in optimization (Example 1).

\begin{tabular}{lcccccccc}
\hline Type & $\alpha_{k}$ & $\beta_{k}$ & $v_{k}$ & $w_{k}$ & $V$ & $C$ & $W$ \\
\hline 1 & $2 \times 10^{-5}$ & 1.5 & 6 & 7 & 160 & 200 & 160 \\
2 & $1 \times 10^{-5}$ & 1.5 & 8 & 8 & & & 1000 \\
\hline
\end{tabular}

TABle 7: Optimal component reliability and swapping strategy (Example 1).

\begin{tabular}{|c|c|c|c|c|c|}
\hline & No. 1 & No. 2 & No. 3 & No. 4 & No. 5 \\
\hline$R_{S}$ & 0.95922268 & 0.95922749 & 0.95923230 & 0.95923213 & 0.95922510 \\
\hline$r_{1}$ & 0.90873718 & 0.90863069 & 0.90847271 & 0.90836202 & 0.90841362 \\
\hline$r_{2}$ & 0.86609207 & 0.86663327 & 0.86742572 & 0.86796996 & 0.86766398 \\
\hline$h_{1}$ & {$\left[\begin{array}{ll}8 & 5\end{array}\right]$} & {$\left[\begin{array}{ll}8 & 5\end{array}\right]$} & {$\left[\begin{array}{ll}8 & 5\end{array}\right]$} & {$\left[\begin{array}{ll}8 & 5\end{array}\right]$} & {$\left[\begin{array}{ll}8 & 5\end{array}\right]$} \\
\hline$h_{2}$ & {$[74 ; 96]$} & {$[74 ; 96]$} & {$[74 ; 96]$} & {$[74 ; 96]$} & {$[74 ; 96]$} \\
\hline Slack (g1) & 30 & 30 & 30 & 30 & 30 \\
\hline Slack (g2) & 0.0173 & 0.0120 & 0.0111 & -0.0028 & 0.0290 \\
\hline Slack (g3) & 29 & 29 & 29 & 29 & 29 \\
\hline
\end{tabular}

TABLE 8: Survival signature of the system under optimal swapping strategy.

\begin{tabular}{cccc}
\hline$l_{1}$ & $l_{2}$ & $\Phi\left(l_{1}, l_{2}\right)$ & $\Phi_{s}\left(l_{1}, l_{2}\right)$ \\
\hline 3 & 2 & $1 / 20$ & $2 / 5$ \\
3 & 3 & $9 / 40$ & $3 / 5$ \\
3 & 4 & $3 / 10$ & $3 / 5$ \\
4 & 1 & $1 / 20$ & $1 / 5$ \\
4 & 2 & $1 / 3$ & $5 / 6$ \\
4 & 3 & $13 / 20$ & 1 \\
4 & 4 & $4 / 5$ & 1 \\
5 & 1 & $1 / 2$ & $3 / 4$ \\
5 & 2 & $5 / 6$ & 1 \\
5 & 3 & 1 & 1 \\
5 & 4 & 1 & 1 \\
\hline
\end{tabular}

means that the current swapping strategy is effective in reliability improving.

Since the component failure time obeys an exponential distribution and the component reliability at time $T$ is already known, we can obtain the reliability of the system at time $t \in[0,1000]$ before and after the optimization using equation (4). Reliability curves are shown in Figure 11, and a comparison of the system and component parameters before and after the optimization is presented in Table 10.
TABLE 9: Importance of components in Example 1.

\begin{tabular}{lc}
\hline Number & Importance \\
\hline 1 & 0.2261 \\
2 & 0.2261 \\
3 & 0.2261 \\
4 & 0.2020 \\
5 & 0.9284 \\
6 & 0.1230 \\
7 & 0.1126 \\
8 & 0.0131 \\
9 & 0.0090 \\
\hline
\end{tabular}

The reliability curves show that the component swapping-based reliability optimization can considerably improve system reliability. Furthermore, the system can still achieve high reliability levels even if the component reliability is lower after the swapping-based optimization, as seen from Table 10, which can reduce unnecessary costs.

Example 2. A system consisting of 16 components of 3 types is shown in Figure 12. Components 1, 4, 7, 10, 12, and 15 are type 1 , components $3,8,11$, and 16 are type 2 , and components $2,5,6,9,13$, and 14 are type 3 . Parameters of the model are listed in Table 11. Similar to Example 1, failure 


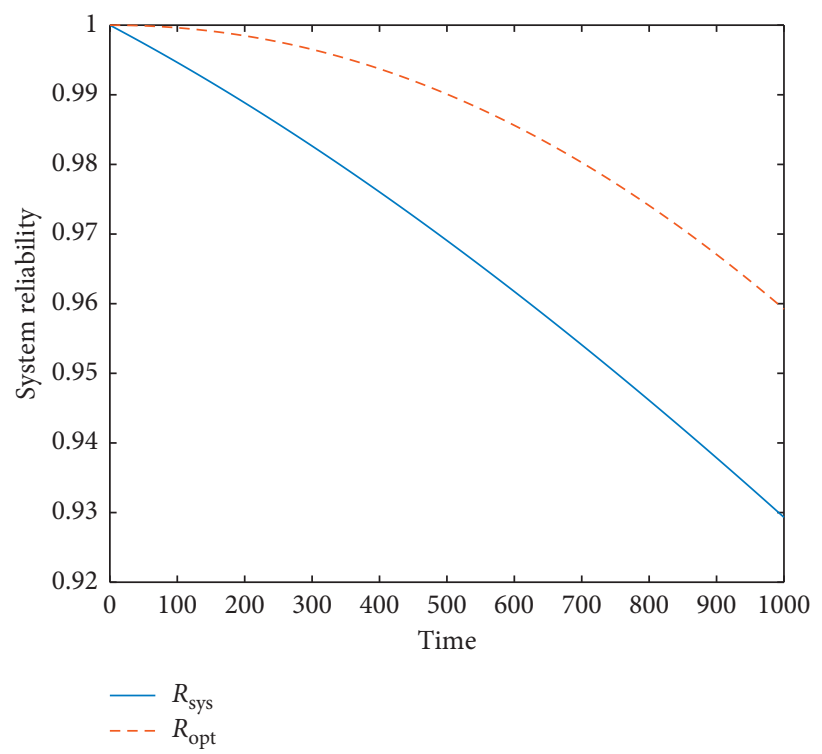

FIGURE 11: System reliability curves (Example 1).

TABLE 10: Comparison of system and component parameters before and after optimization.

\begin{tabular}{lccc}
\hline System reliability & Component reliability & Component swapping & Cost \\
\hline 0.92932008 & $r 1=0.950$ & None & 369 \\
$(t=T)$ & $r 2=0.930$ & {$\left[\begin{array}{ccc}8 & 5\end{array}\right]$} & \\
0.95923230 & $r 1=0.908$ & {$\left[\begin{array}{lll}7 & 4 ; 9 & 6\end{array}\right]$} & 200 \\
$(t=T)$ & $r 2=0.867$ & & \\
\hline
\end{tabular}

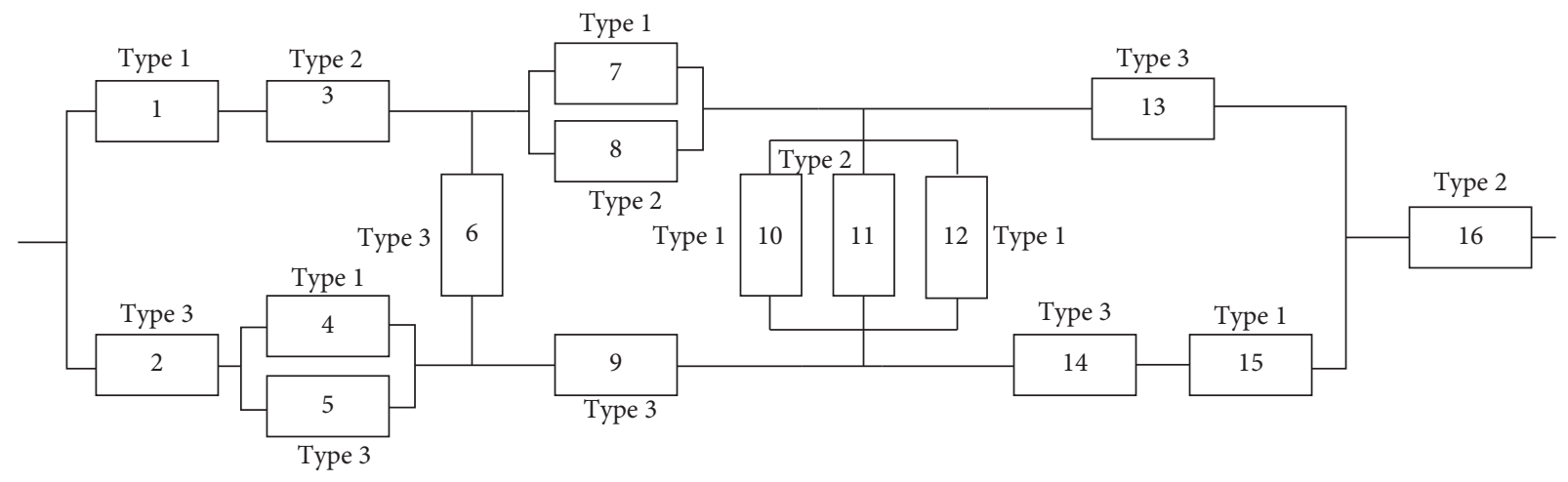

Figure 12: System with 16 components of 3 types.

times of the components in the system are assumed to obey an exponential distribution, and failure rates of each component, type 1 , type 2 , and type 3 , at time Tare $0.06,0.03$, and 0.02 , respectively.

The replacement-based reliability optimization mathematical model for Example 2 can be described as follows:
TABle 11: Parameters used in optimization (Example 2).

\begin{tabular}{lcccccccc}
\hline Type & $\alpha_{k}$ & $\beta_{k}$ & $v_{k}$ & $w_{k}$ & $V$ & $C$ & $W$ & $T$ \\
\hline 1 & $2 \times 10^{-5}$ & 1.5 & 6 & 7 & 300 & 320 & 350 & 1000 \\
2 & $1 \times 10^{-5}$ & 1.5 & 8 & 8 & & & & \\
3 & $1 \times 10^{-5}$ & 1.5 & 7 & 8 & & & & \\
\hline
\end{tabular}


TABLE 12: Optimal component reliability and swapping strategy (Example 2).

\begin{tabular}{|c|c|c|c|c|c|}
\hline Component no. & 1 & 2 & 3 & 4 & 5 \\
\hline$R_{S}$ & 0.99201627 & 0.99218370 & 0.99218370 & 0.99208033 & 0.99218370 \\
\hline$r_{1}$ & 0.81578079 & 0.82219070 & 0.82219070 & 0.81904807 & 0.82219070 \\
\hline$r_{2}$ & 0.93593029 & 0.93655845 & 0.93655845 & 0.93462883 & 0.93655845 \\
\hline$r_{3}$ & 0.91029170 & 0.90963046 & 0.90963046 & 0.91438079 & 0.90963046 \\
\hline$h_{1}$ & [10 1; 12 7] & {$[101 ; 127]$} & {$[101 ; 127]$} & {$[101 ; 127]$} & [10 $1 ; ; 12$ 7] \\
\hline$h_{2}$ & {$\left[\begin{array}{llll}11 & 16 ; 8 & 3\end{array}\right]$} & {$\left[\begin{array}{lll}11 & 16 ; & 8\end{array}\right]$} & {$\left[\begin{array}{lllll}11 & 16 ; & 8 & 3\end{array}\right]$} & {$[1116 ; 8$ 3 $]$} & {$\left[\begin{array}{llll}11 & 16 ; & 8 & 3\end{array}\right]$} \\
\hline$h_{3}$ & {$[613 ; 142]$} & {$[513 ; 142]$} & {$[513 ; 142]$} & {$[513 ; 62]$} & {$[513 ; 142]$} \\
\hline Slack (g1) & 32 & 32 & 32 & 32 & 32 \\
\hline Slack (g2) & 5.7331 & 0.7416 & 0.7416 & 0.0363 & 0.7416 \\
\hline Slack (g3) & 66 & 66 & 66 & 66 & 66 \\
\hline
\end{tabular}

TABLE 13: Importance of components in Example 2.

\begin{tabular}{lc}
\hline Number & Importance \\
\hline 1 & 0.0960 \\
2 & 0.2058 \\
3 & 0.0843 \\
4 & 0.0172 \\
5 & 0.0338 \\
6 & 0.0177 \\
7 & 0.0054 \\
8 & 0.0152 \\
9 & 0.0269 \\
10 & 0.0001 \\
11 & 0.0004 \\
12 & 0.0001 \\
13 & 0.2299 \\
14 & 0.0677 \\
15 & 0.0749 \\
16 & 0.9509 \\
\hline
\end{tabular}

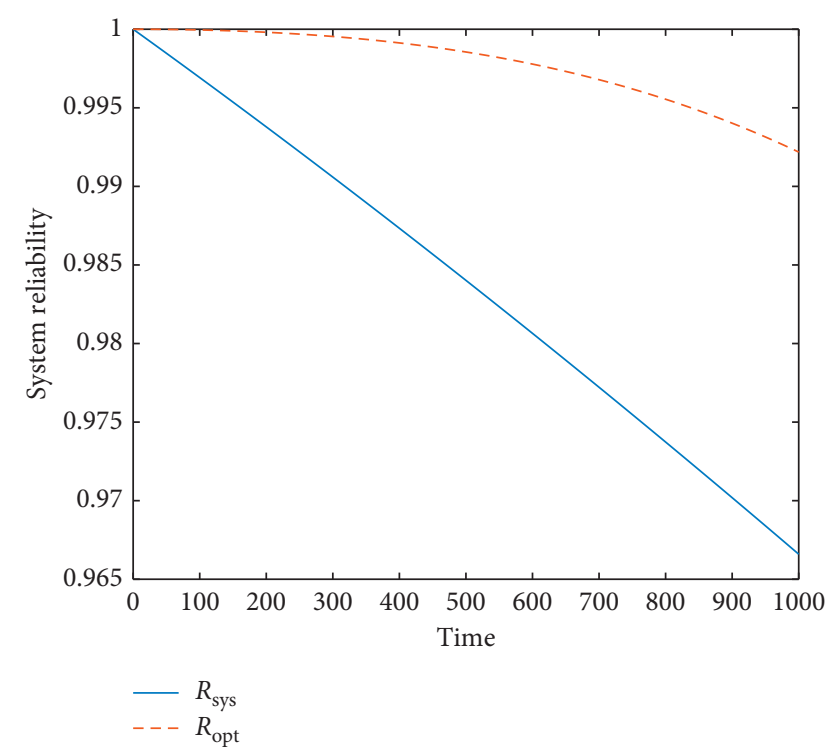

Figure 13: System reliability curves (Example 2).
TABLE 14: Comparison of parameters before and after optimization (Example 2).

\begin{tabular}{lccc}
\hline $\begin{array}{l}\text { System } \\
\text { reliability }\end{array}$ & $\begin{array}{c}\text { Component } \\
\text { reliability }\end{array}$ & $\begin{array}{c}\text { Component } \\
\text { swapping }\end{array}$ & Cost \\
\hline 0.96659353 & $r 1=0.940$ & & \\
$(t=T)$ & $r 2=0.970$ & None & 463 \\
& $r 3=0.980$ & & \\
0.99218370 & $r 1=0.822$ & {$\left[\begin{array}{lll}10 & 1 ; 12 & 7\end{array}\right]$} & \\
$(t=T)$ & $r 2=0.937$ & {$[1116 ; 8$} & 319 \\
& $r 3=0.910$ & {$\left[\begin{array}{lll}5 & 13 ; 14\end{array}\right]$} & \\
\hline
\end{tabular}

$$
\begin{aligned}
\text { maximum } \quad R_{\mathrm{s}}(\mathbf{r}, \mathbf{h}) & =\sum_{l_{1}=0}^{6} \sum_{l_{2}=0}^{4} \sum_{l_{3}=0}^{6}\left[\Phi_{h_{1}, h_{2}, h_{3}}\left(l_{1}, l_{2}, l_{3}\right) \prod_{k=1}^{3} r_{k}^{l_{k}}\left(1-r_{k}\right)^{m_{k}-l_{k}}\right], \\
\text { subject to } \quad g_{1}(\mathbf{r}, \mathbf{h}) & =\sum_{k=1}^{3}\left(v_{k} n_{k}^{3}+v_{k} m_{k}\right)-V \leq 0, \\
g_{2}(\mathbf{r}, \mathbf{h}) & =\sum_{k=1}^{3} \alpha_{k}\left(-\frac{T}{\ln \left(r_{k}\right)}\right)^{\beta_{k}}\left[m_{k}+n_{k}+\exp \left(\frac{n_{k}}{4}\right)\right]-C \leq 0, \\
g_{3}(\mathbf{r}, \mathbf{h}) & =\sum_{k=1}^{3}\left(\omega_{k} n_{k}^{3}+\omega_{k} m_{k}\right)-W \leq 0 .
\end{aligned}
$$

The maximum number of cycles of the algorithm was set to 4000 , the population of solutions was set to 50 , and the limit update number was set to 100 . Then, the optimization was performed by applying the penalty guided ABC algorithm. Results for the optimal component reliability and swapping strategy are presented in Table 12.

After multiple calculations, the optimal reliabilities of each type of component at time $t=T$ were 0.82219070 , 0.93655845 , and 0.90963046 . The optimal swapping strategy for the system is as follows: component 10 is set to replace component 1 and component 12 is set to replace component 7 in type 1 ; component 11 is set to replace component 16 and component 8 is set to replace component 3 in type 2 ; component 5 is set to replace 


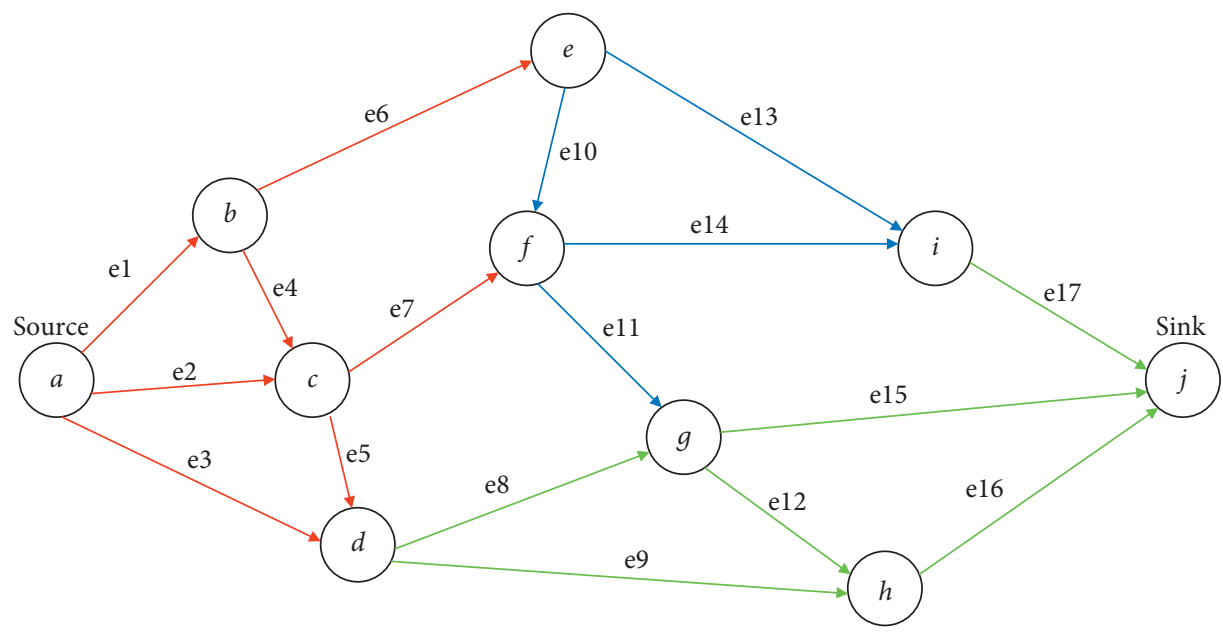

FIgURE 14: Network system with 10 edges and 17 vertices.

TABle 15: Parameters used in optimization (Example 3).

\begin{tabular}{lccccccr}
\hline Type & $\alpha_{k}$ & $\beta_{k}$ & $v_{k}$ & $w_{k}$ & $V$ & $C$ & $W$ \\
\hline 1 & $1.5 \times 10^{-5}$ & 1.5 & 5 & 3 & 350 & 250 & 300 \\
2 & $2 \times 10^{-5}$ & 1.5 & 7 & 4 & & & 1000 \\
3 & $1 \times 10^{-5}$ & 1.5 & 7 & 6 & & \\
\hline
\end{tabular}

TABLE 16: Optimal component reliability and swapping strategy (Example 3).

\begin{tabular}{|c|c|c|c|c|c|}
\hline Component no. & 1 & 2 & 3 & 4 & 5 \\
\hline$R_{S}$ & 0.99975193 & 0.99974988 & 0.99975193 & 0.99975193 & 0.99975193 \\
\hline$r_{1}$ & 0.89355334 & 0.89355334 & 0.89355334 & 0.89355334 & 0.89355334 \\
\hline$r_{2}$ & 0.80957855 & 0.80731652 & 0.80957855 & 0.80957855 & 0.80957855 \\
\hline$r_{3}$ & 0.91690166 & 0.91690166 & 0.91690166 & 0.91690166 & 0.91690166 \\
\hline$h_{1}$ & {$[43 ; 67 ; 52]$} & {$[43 ; 67 ; 52]$} & {$[43 ; 67 ; 52]$} & {$[43 ; 67 ; 52]$} & {$[43 ; 67 ; 52]$} \\
\hline$h_{2}$ & {$\left[\begin{array}{ll}10 & 11\end{array}\right]$} & {$\left[\begin{array}{ll}10 & 11\end{array}\right]$} & {$\left[\begin{array}{ll}10 & 11\end{array}\right]$} & {$\left[\begin{array}{ll}10 & 11\end{array}\right]$} & {$\left[\begin{array}{ll}10 & 11\end{array}\right]$} \\
\hline$h_{3}$ & {$\left[\begin{array}{llll}12 & 16 ; & 8 & 15\end{array}\right]$} & {$\left[\begin{array}{llll}12 & 16 ; 8 & 15\end{array}\right]$} & {$\left[\begin{array}{llll}12 & 16 ; & 8 & 15\end{array}\right]$} & {$\left[\begin{array}{llll}12 & 16 ; & 8 & 15\end{array}\right]$} & {$\left[\begin{array}{llll}12 & 16 ; & 8 & 15\end{array}\right]$} \\
\hline Slack (g1) & 7 & 7 & 7 & 7 & 7 \\
\hline Slack (g2) & 0.0005 & 0.8005 & 0.0005 & 0.0005 & 0.0005 \\
\hline Slack (g3) & 4 & 4 & 4 & 4 & 4 \\
\hline
\end{tabular}

TABLE 17: Importance of components in Example 3.

\begin{tabular}{lc}
\hline Number & Importance \\
\hline 1 & 0.0161 \\
2 & 0.0174 \\
3 & 0.0210 \\
4 & 0.0015 \\
5 & 0.0049 \\
6 & 0.0061 \\
7 & 0.0074 \\
8 & 0.0092 \\
9 & 0.0056 \\
10 & 0.0010 \\
11 & 0.0043 \\
12 & 0.0012 \\
13 & 0.0027 \\
14 & 0.0039 \\
15 & 0.0148 \\
16 & 0.0181 \\
17 & 0.0129 \\
\hline
\end{tabular}

component 13 and component 14 is set to replace component 2 in type 3 . Importance of components is listed in Table 13 which shows that importance of target components is higher than that of spare components in each type. Reliability curves of the system before and after the optimization are shown in Figure 13. A comparison of the system and component parameters before and after the optimization is presented in Table 14.

Example 3. An enterprise core directed network system is shown in Figure 14. The condition for the system to remain functioning is that the source and sink vertices are connected by at least one path. It is assumed that all vertices are reliable, and the edges are of three types. Type 1 consists of e1, e2, e3, e4, e5, e6, and e7 with failure rate 0.08 at time $T$; type 2 consists of e10, e11, e13, and e14 with failure rate 0.07 at time $T$; type 3 consists of e8, e9, e12, e15, e16, and e17 with failure rate 0.05 at time $T$. The failure times of the edges are all 


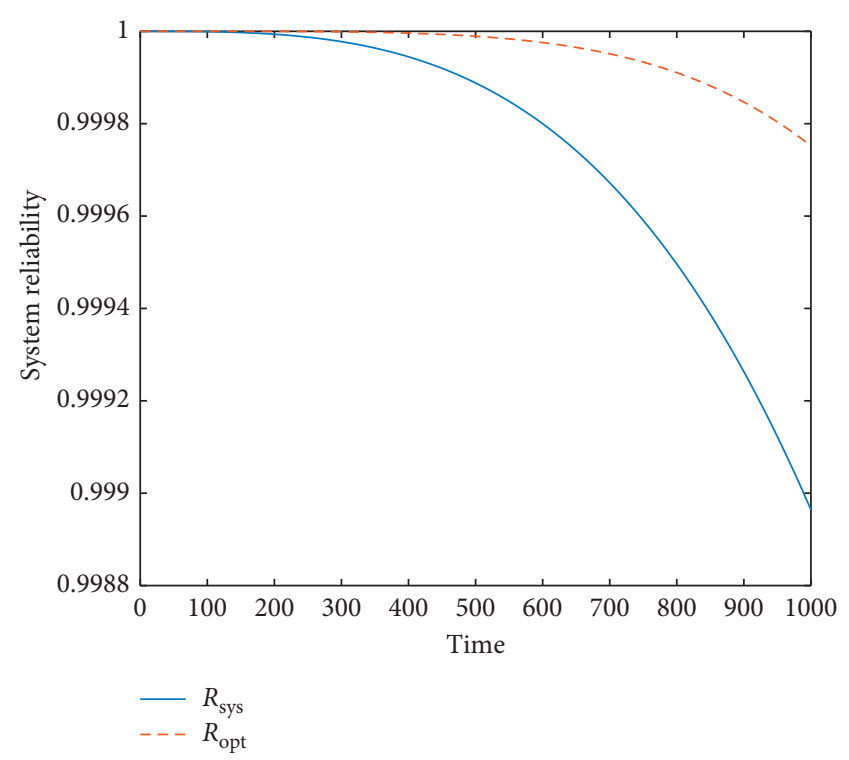

Figure 15: System reliability curves (Example 3).

TABLE 18: Comparison of parameters before and after optimization (Example 3).

\begin{tabular}{lccc}
\hline $\begin{array}{l}\text { System } \\
\text { reliability }\end{array}$ & $\begin{array}{c}\text { Component } \\
\text { reliability }\end{array}$ & $\begin{array}{c}\text { Component } \\
\text { swapping }\end{array}$ & Cost \\
\hline 0.99896407 & $r 1=0.920$ & & \\
$(t=T)$ & $r 2=0.930$ & None & 312 \\
& $r 3=0.950$ & & \\
0.99975193 & $r 1=0.893$ & {$[43 ; 67 ; 52$} & \\
$(t=T)$ & $r 2=0.809$ & {$[1011]$} & 250 \\
& $r 3=0.916$ & {$[1216 ; 815]$} & \\
\hline
\end{tabular}

assumed to obey exponential distribution. Parameters used in the model are listed in Table 15.

The replacement-based reliability optimization mathematical model for Example 3 can be described as follows:

$$
\begin{aligned}
\text { maximum } \quad R_{s}(\mathbf{r}, \mathbf{h}) & =\sum_{l_{1}=0}^{7} \sum_{l_{2}=0}^{4} \sum_{l_{3}=0}^{6}\left[\Phi_{h_{1}, h_{2}, h_{3}}\left(l_{1}, l_{2}, l_{3}\right) \prod_{k=1}^{3} r_{k}^{l_{k}}\left(1-r_{k}\right)^{m_{k}-l_{k}}\right], \\
\text { subject to } \quad g_{1}(\mathbf{r}, \mathbf{h}) & =\sum_{k=1}^{3}\left(v_{k} n_{k}^{3}+v_{k} m_{k}\right)-V \leq 0, \\
g_{2}(\mathbf{r}, \mathbf{h}) & =\sum_{k=1}^{3} \alpha_{k}\left(-\frac{T}{\ln \left(r_{k}\right)}\right)^{\beta_{k}}\left[m_{k}+n_{k}+\exp \left(\frac{n_{k}}{4}\right)\right]-C \leq 0, \\
g_{3}(\mathbf{r}, \mathbf{h}) & =\sum_{k=1}^{3}\left(\omega_{k} n_{k}^{3}+\omega_{k} m_{k}\right)-W \leq 0 .
\end{aligned}
$$

The maximum number of cycles of the algorithm was set to 5000 , the population of solutions was set to 50 , and the limit update number was set to 100 . Then, the optimization was performed by applying the penalty guided $\mathrm{ABC}$ algorithm. Results for the optimal component reliability and swapping strategy are presented in Table 16.
After multiple calculations, the optimal reliabilities of each type of component at time $t=T$ were 0.89355334 , 0.80957855 , and 0.91690166 . The optimal swapping strategy for the system is as follows: component 4 is set to replace component 3, component 6 is set to replace component 7 , and component 5 is set to replace component 2 in type 1 ; component 10 is set to replace component 11 in type 2; component 12 is set to replace component 16 and component 8 is set to replace component 15 in type 3 . Importance of components (edges) is listed in Table 17 which shows that importance of target components is higher than that of spare components in each type. Reliability curves of the network system before and after the optimization are shown in Figure 15. A comparison of the system and component parameters before and after the optimization is presented in Table 18.

\section{Conclusions}

In this paper, a new system reliability optimization model based on component swapping is proposed to achieve the optimal component reliability and to determine the best swapping strategy for each type of component. The analysis was performed under certain constraint functions for volume, weight, and cost. The survival signature and truth table are introduced into the system reliability calculations, by which the structure of the system can be expressed as a matrix, and the system reliability under different swapping strategies can be calculated more efficiently using matrix operations.

The artificial bee colony algorithm is applied to solve this type of optimization problem. The solution initialization and local search of the original $\mathrm{ABC}$ are modified using matrix operations for component swapping. Three swapping-based system reliability optimization examples are established, and optimizations were performed using the $\mathrm{ABC}$ algorithm. The results show that the component swapping-based optimization can effectively improve system reliability within limited costs.

It should be noted that the component-swapping strategy discussed in this paper is limited to a one-to-one component pattern, whereas in practice, swapping strategies can be more flexible. For example, more than one component could potentially be used to replace an important component if necessary, making the system more robust. The component swapping proposed in this paper is timeconsuming which would be impossible to achieve in practice. The time required for swapping operations can be viewed as a factor affecting the system reliability and optimization costs and will therefore be considered in future work. Furthermore, the proposed swapping-based optimization could be combined with redundancy allocation to form a new system reliability optimization model for determining the optimal redundancy level and componentswapping strategy.

\section{Data Availability}

The data used to support the findings of this study are available from the corresponding author upon request. 


\section{Conflicts of Interest}

The authors declare that they have no conflicts of interest.

\section{Acknowledgments}

The authors gratefully acknowledge the support of the National Natural Science Foundation of China (51975110, 51575094, and 51875094).

\section{References}

[1] K. Kołowrocki and S. B. Kwiatuszewska, "Reliability and risk analysis of large systems with ageing components," Reliability Engineering and System Safety, vol. 93, no. 12, pp. 1821-1829, 2008.

[2] E. Zio, "Reliability engineering: old problems and new challenges," Reliability Engineering \& System Safety, vol. 94, no. 2, pp. 125-141, 2009.

[3] X. Huang, Y. Li, Y. Zhang, and X. Zhang, "A new direct second-order reliability analysis method," Applied Mathematical Modelling, vol. 55, pp. 68-80, 2018.

[4] J. L. Qin and Z. Zheng, "Reliability and sensitivity analysis method for a multistate system with common cause failure," Complexity, vol. 2019, Article ID 6535726, 8 pages, 2019.

[5] W. Kuo and V. R. Prasad, "An annotated overview of systemreliability optimization," IEEE Transactions on Reliability, vol. 49, no. 2, pp. 176-187, 2000.

[6] W. Kwo and R. Wan, "Recent advances in optimal reliability allocation," IEEE Transactions on reliability, Man and Cybernetics, vol. 37, no. 2, pp. 143-156, 2007.

[7] C. Ha and W. Kuo, "Reliability redundancy allocation: an improved realization for nonconvex nonlinear programming problems," European Journal of Operational Research, vol. 171, no. 1, pp. 24-38, 2006.

[8] O. Abedinia, N. Amjady, and A. Ghasemi, "A new metaheuristic algorithm based on shark smell optimization," Complexity, vol. 21, no. 5, pp. 97-116, 2016.

[9] M. A. Mellal and E. Zio, "A penalty guided stochastic fractal search approach for system reliability optimization," Reliability Engineering \& System Safety, vol. 152, pp. 213-227, 2016.

[10] T. Y. Zheng and W. L. Luo, "An improved squirrel search algorithm for optimization,” Complexity, vol. 2019, Article ID 6291968, 2019.

[11] H. Kim and P. Kim, "Reliability-redundancy allocation problem considering optimal redundancy strategy using parallel genetic algorithm," Reliability Engineering \& System Safety, vol. 159, pp. 153-160, 2017.

[12] A. Kumar, S. Pant, and M. Ram, "System reliability optimization using gray wolf optimizer algorithm," Quality and Reliability Engineering International, vol. 33, no. 7, pp. 1327-1335, 2017.

[13] M. Caserta and S. Voss, "An exact algorithm for the reliability redundancy allocation problem," European Journal of Operational Research, vol. 244, no. 1, pp. 110-116, 2015.

[14] M. Hemmati, M. Amiri, and M. Zandieh, "Optimization redundancy allocation problem with nonexponential repairable components using simulation approach and artificial neural network," Quality and Reliability Engineering International, vol. 34, no. 3, pp. 278-297, 2018.

[15] X. Huang, F. P. A. Coolen, and T. Coolen-Maturi, "A heuristic survival signature based approach for reliability-redundancy allocation," Reliability Engineering \& System Safety, vol. 185, pp. 511-517, 2019.
[16] N. Wang, J. B. Zhao, Z. Y. Jiang, and S. Zhang, "Reliability optimization of systems with component improvement cost based on importance measure," Advances in Mechanical Engineering, vol. 10, no. 11, 2018.

[17] Y. Gao, F. Zhang, and Y. Li, "Reliability optimization design of a planar multi-body system with two clearance joints based on reliability sensitivity analysis," Proceedings of the Institution of Mechanical Engineers, Part C: Journal of Mechanical Engineering Science, vol. 233, no. 4, pp. 1369-1382, 2019.

[18] J. K. Chen, Y. He, and W. Wei, "Reliability analysis and optimization of equal load-sharing k-out-of-n phased-mission systems," Eksploatacja i Niezawodnosc-Maintenance and Reliability, vol. 17, no. 2, pp. 250-259, 2015.

[19] S. H. Chung, H. C. W. Lau, G. T. S. Ho, and W. H. Ip, "Optimization of system reliability in multi-factory production networks by maintenance approach," Expert Systems with Applications, vol. 36, no. 6, pp. 10188-10196, 2009.

[20] I. J. Ramirez-Rosado and J. L. Bernal-Agustin, "Reliability and costs optimization for distribution networks expansion using an evolutionary algorithm," IEEE Transactions on Power Systems, vol. 16, no. 1, pp. 111-118, 2001.

[21] R. Cao, W. Hou, and Y. Gao, "An entropy-based three-stage approach for multi-objective system reliability optimization considering uncertainty," Engineering Optimization, vol. 50, no. 9, pp. 1453-1469, 2018.

[22] P. K. Muhuri, Z. Ashraf, and Q. M. D. Lohani, "Multi objective reliability redundancy allocation problem with interval type-2 fuzzy uncertainty," IEEE Transactions on Fuzzy Systems, vol. 26, no. 3, pp. 1339-1355, 2018.

[23] A. Najem and F. P. A. Coolen, "System reliability and component importance when components can be swapped upon failure," Applied Stochastic Models in Business and Industry, vol. 35, no. 3, pp. 399-413, 2019.

[24] W. Kuo and X. Zhu, Importance Measures in Reliability, Risk, and Optimization: Principles and Applications, Wiley, Chichester, UK, 2012.

[25] F. J. Samaniego, System Signatures and Their Applications in Engineering Reliability, Springer, New York, NY, USA, 2007.

[26] F. P. A. Coolen and T. Coolen-Maturi, "On the structure function and survival signature for system reliability," Safety and Reliability, vol. 36, no. 2, pp. 77-87, 2016.

[27] R. G. Bennetts, "Analysis of reliability block diagrams by boolean techniques," IEEE Transactions on Reliability, vol. 31, no. 2, pp. 159-166, 1982.

[28] F. P. A. Coolen and T. Coolen-Maturi, "Generalizing the signature to system with multiple types of components," Complex Systems and Dependability, vol. 170, pp. 115-130, 2012.

[29] L. J. M. Aslett, F. P. A. Coolen, and S. P. Wilson, "Bayesian inference for reliability of systems and networks using the survival signature," Risk Analysis, vol. 35, no. 9, pp. 16401651, 2015.

[30] Z. Birnbaum, "On the importance of different components in a multicomponent system," Multivariate Analysis, vol. 3, no. 1, pp. 581-592, 1969.

[31] D. Karaboga and B. Basturk, "A powerful and efficient algorithm for numerical function optimization: artificial bee colony (ABC) algorithm," Journal of Global Optimization, vol. 39, no. 3, pp. 459-471, 2007.

[32] W.-C. Yeh and T.-J. Hsieh, "Solving reliability redundancy allocation problems using an artificial bee colony algorithm," Computers \& Operations Research, vol. 38, no. 11, pp. 14651473, 2011. 


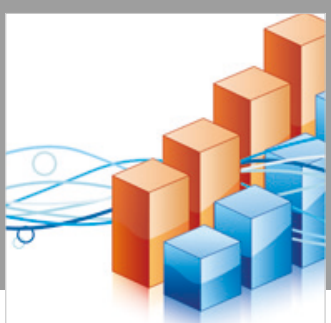

Advances in

Operations Research

\section{-n-m}
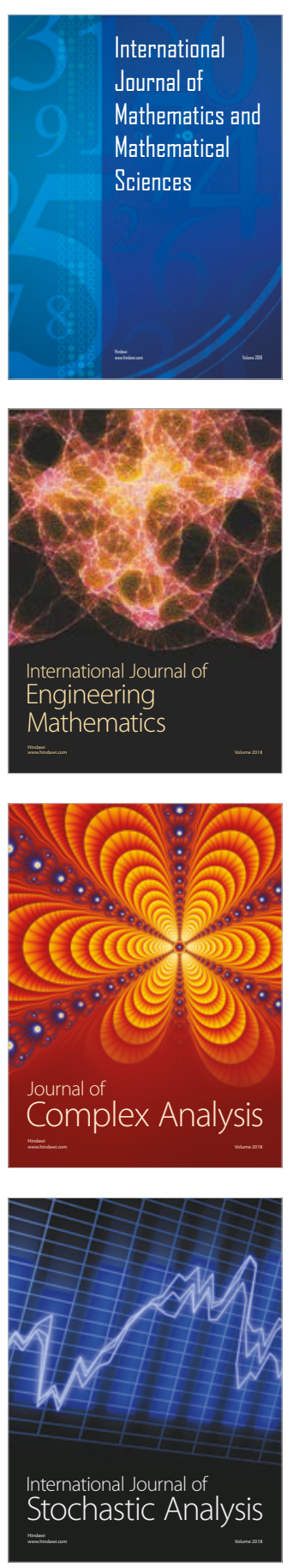
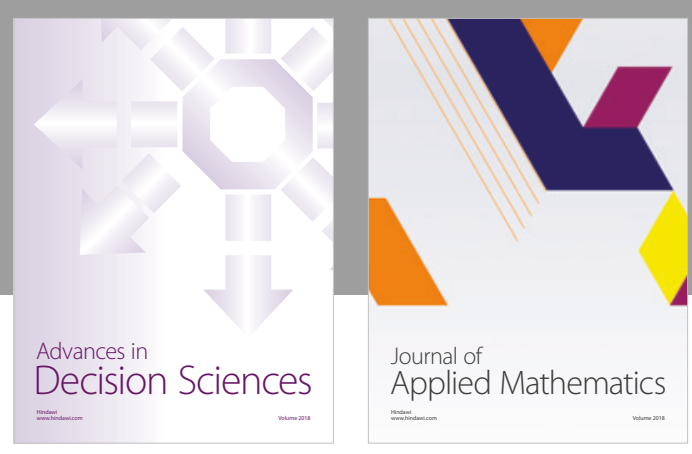

Journal of

Applied Mathematics
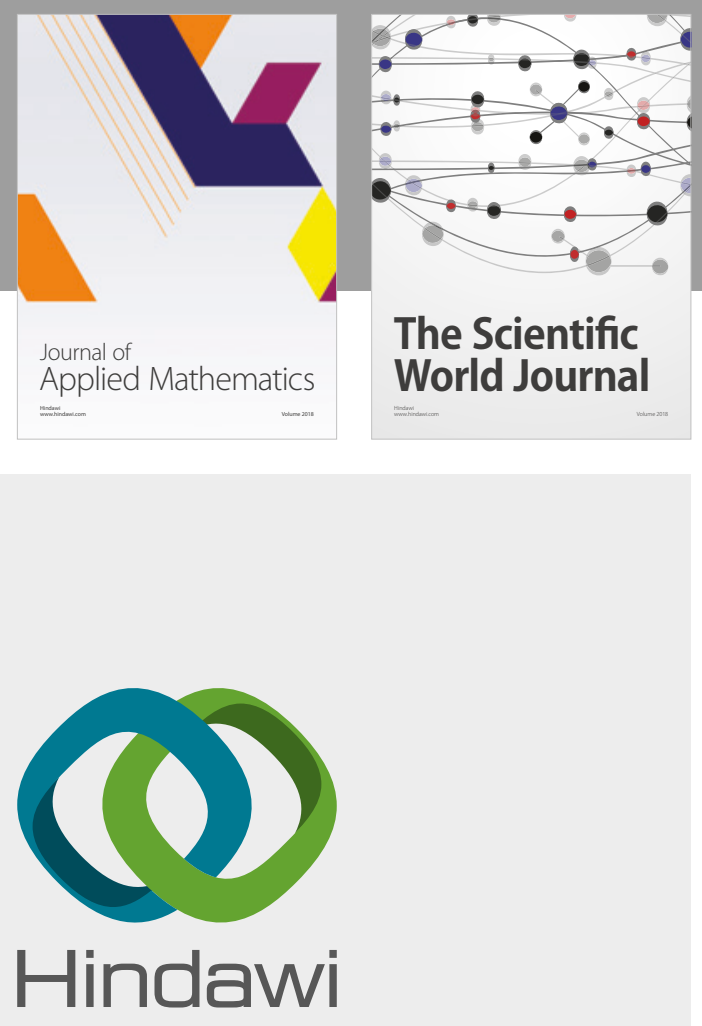

Submit your manuscripts at

www.hindawi.com

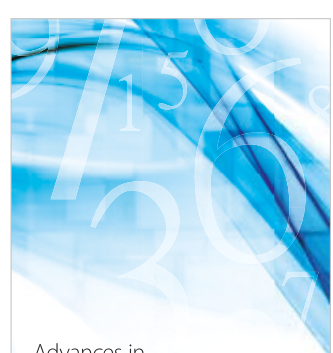

Advances in
Numerical Analysis
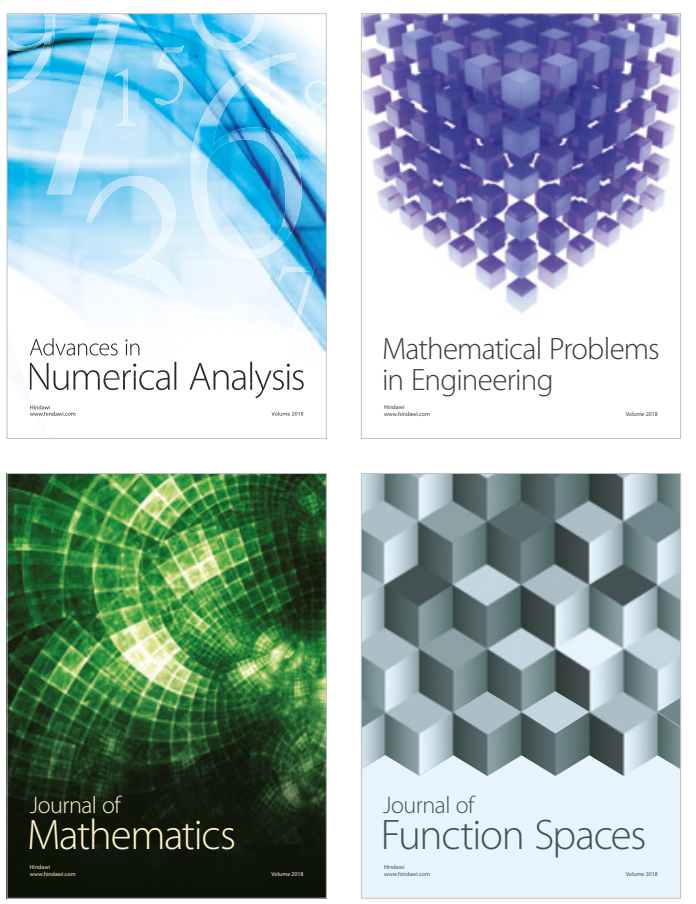

Mathematical Problems in Engineering

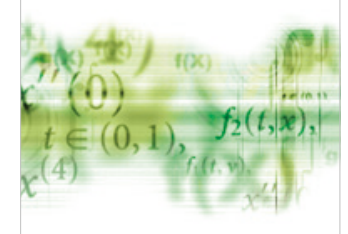

International Journal of

Differential Equations

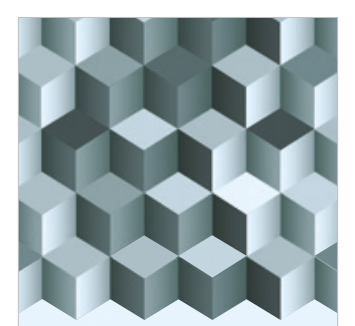

Journal of

Function Spaces
The Scientific

World Journal

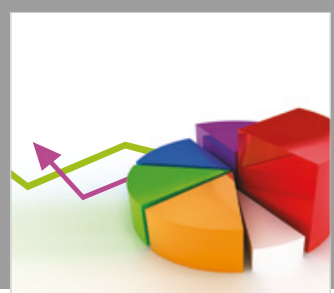

Journal of

Probability and Statistics
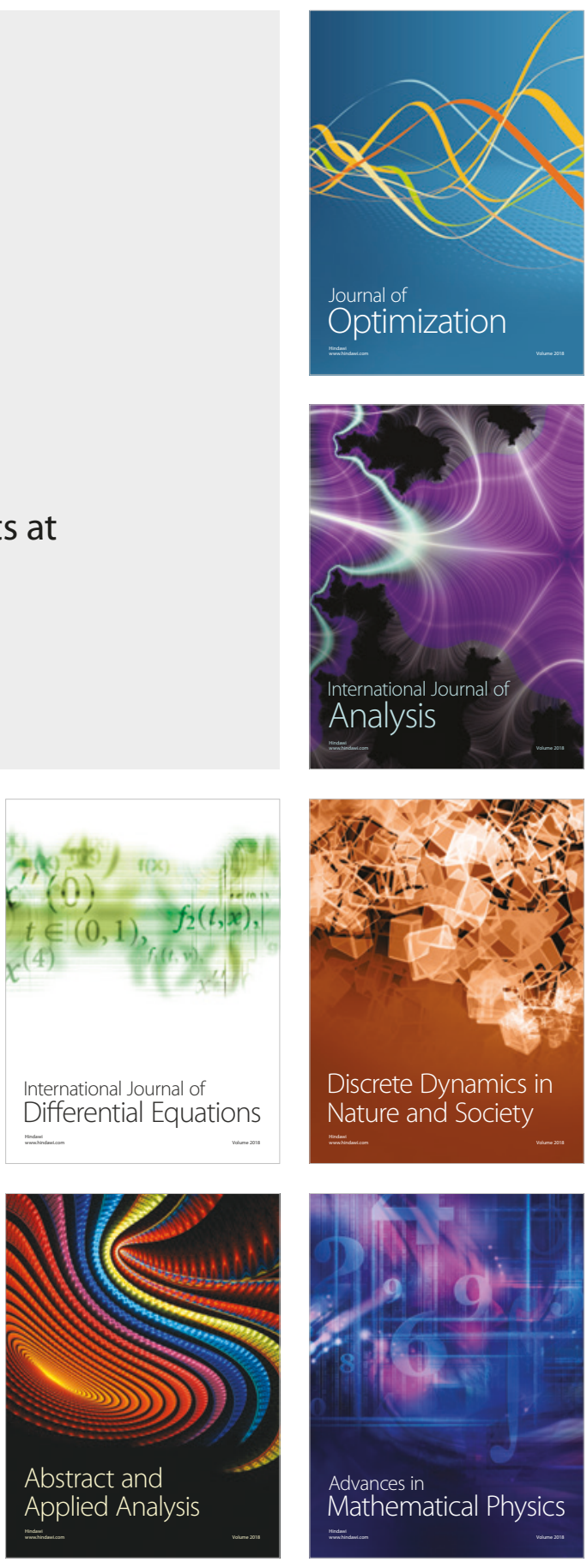